associated with the gut microbiota even after adjusting for the country, age and sex (PERMANOVA, Pseudo-F=4.206, $\left.\mathrm{R}^{2}=0.019, \mathrm{p}=0.001\right)$. Several taxa were found to be differentially abundant across ethnicity (ANCOM-BC, q<0.05). Notably, Ligilactobacillus, a lactic acid bacteria, was elevated among Indians (IDDF2021-ABS-0150 Figure 2. Ancom.ci) and reduced among Malay (IDDF2021-ABS-0150 Figure 3. Ancom.cm) relative to Chinese, suggesting that differences in the dietary pattern were responsible for the observed gut variation. Jakun exhibited the most differentially abundant gut microbiota (IDDF2021-ABS-0150 Figure 4. Ancom), with an elevated abundance of 18, 6, 5, and 1 genera, families, order, and class, respectively. The higher diversity of the Jakun was likely a reflection of their more traditional

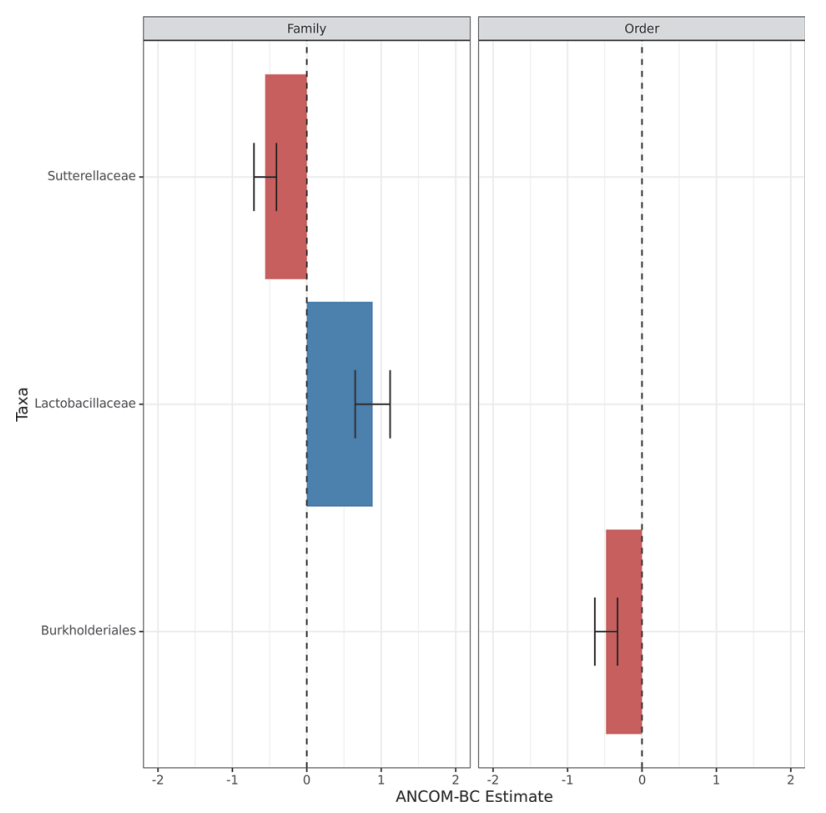

Abstract IDDF2021-ABS-0150 Figure 2

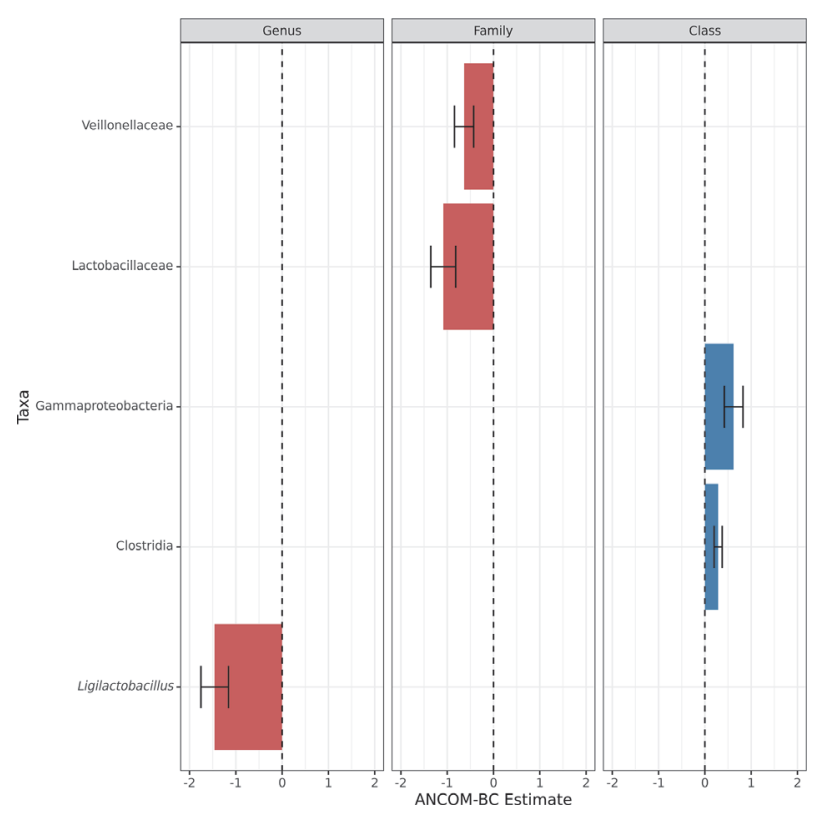

Abstract IDDF2021-ABS-0150 Figure 3

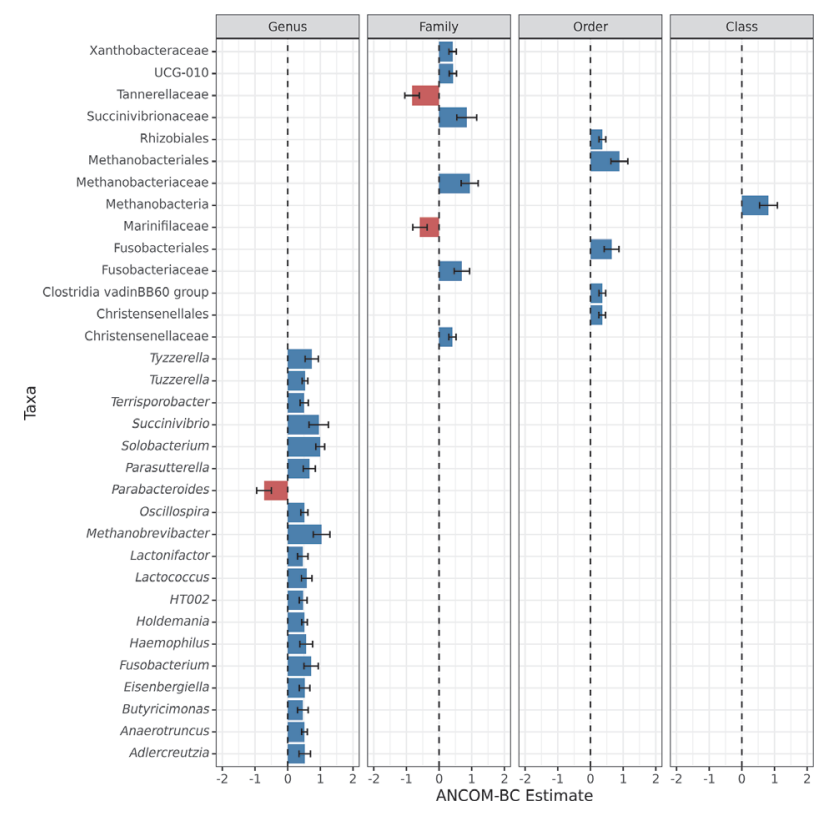

Abstract IDDF2021-ABS-0150 Figure 4

way of life, which has been associated with better gut diversity and health, compared to the Chinese, Indian and Malay of both countries, in particular, the higher abundance of Methanobacteria in Jakun has been inversely associated with irritable bowel syndrome.

Conclusions Gut-ethnic differences persist across geographical regions, which was likely due to similar lifestyle and cultural practices by individuals sharing similar ancestry. These signals provide potential biomarkers on the role of the gut microbiota in the aetiology of the unequal disease burdens affecting the different ethnic groups in this multicultural region.

\section{IDDF2021-ABS-0164 GUT FEELINGS IN DEPRESSION: MICROBIOTA DYSBIOSIS IN RESPONSE TO ANTIDEPRESSANTS}

${ }^{1}$ Vengadesh Letchumanan*, 'Angel Yun-Kuan Thye, ${ }^{2}$ Loh Teng-Hern Tan, ${ }^{1}$ Jodi WoanFei Law, 'Dinyadarshini Johnson, 'Hooi-Leng Ser, ${ }^{3}$ Saatheeyavaane Bhuvanendran, ${ }^{2}$ Sivakumar Thurairajasingam, 'Learn-Han Lee. 'Novel Bacteria and Drug Discovery Research Group (NBDD), Microbiome and Bioresource Research Strength (MBRS), Jeffrey Cheah School of Medicine and Health Sciences, Monash University Malaysia, Malaysia; ${ }^{2}$ Clinical School Johor Bahru, Jeffrey Cheah School of Medicine and Health Sciences, Monash University Malaysia, Malaysia; ${ }^{3}$ Brain Research Institute of Monash Sunway (BRIMS), Jeffrey Cheah School of Medicine and Health Sciences, Monash University Malaysia, Malaysia

\subsection{6/gutjil-2021-IDDF.47}

Background Antidepressants are a lifesaver for many people worldwide, regardless of their age or gender. Antidepressant therapy has been the choice for patients with depression, anxiety, and schizophrenia. The gut-brain axis (GBA) is a bidirectional pathway illustrating the communication between the brain and the gut microbiota and vice versa. Many studies have demonstrated the establishing of gut dysbiosis status in major depressive disorder. Meanwhile, the impact of antidepressant treatments on gut microbiota composition remains underexplored. Interestingly, several classes of antidepressants drugs, including monoamine oxidase inhibitors (MAOIs), selective serotonin reuptake inhibitors (SSRIs), N-methyl-d-aspartate 


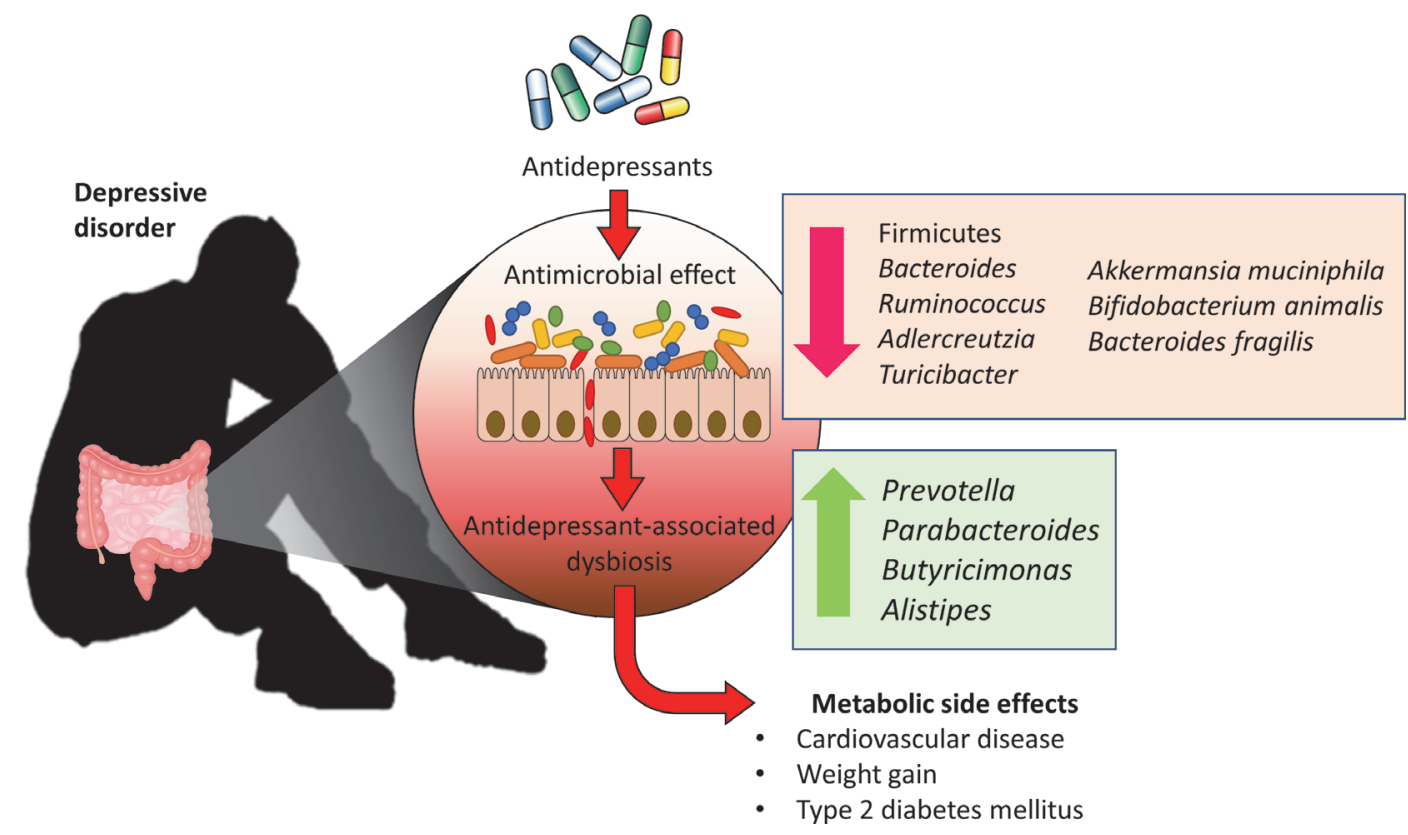

Abstract IDDF2021-ABS-0164 Figure 1

(NMDA) receptor antagonists, and tricyclic antidepressants (TCAs), exhibit antibacterial activities. Hence, this systematic review explores the impact of antidepressants on gut microbiota and potential strategies to alleviate antidepressant-associated dysbiosis.

Methods This systematic review was conducted based on the PRISMA guidelines. Predefined MeSH terms 'antidepressants AND gut microbiome'; 'antidepressants AND antimicrobial activity' were used in three databases (Pubmed, Embase, ProQuest; from database inception to June 2021). Studies reporting on the gut microbiota variation and antidepressants action were included. Studies without antidepressants and/or gut microbiome data were excluded, including conference proceedings, reviews, systematic reviews, meta-analyses, and commentaries.

Results According to the study's inclusion criteria, twelve studies out of 300 articles were selected for the qualitative analysis. In the in-vivo studies, animals administrated with SSRIs had a decreased abundance of Firmicutes, Alphaproteobacteria, Bacteroides, Ruminococcus, Adlercreutzia, Turicibacter, and alpha diversity but an increase in Prevotella, Parabacteroides, Butyricimonas, and Alistipes. Besides, antidepressants were shown to exhibit significant in vitro antimicrobial activity against Akkermansia muciniphila, Bifidobacterium animalis, and Bacteroides fragilis, suggesting that the chronic use of antidepressants potentially causes adverse effects due to their antimicrobial effects and dysbiosis (IDDF2021-ABS-0164 Figure 1. Illustration of gut microbiota dysbiosis in response to antidepressants). Probiotics, prebiotics and fecal microbiota transplantation are shown to be promising strategies to ameliorate antidepressant-associated dysbiosis.

Conclusions Understanding the interaction between antidepressants and gut microbiota, including dysbiosis as a consequence of treatment and potential side effects, is vital for the future development of better and personalized treatment. Supplementing the gut microbiome with prebiotics/probiotics could be an adjuvant treatment to improve the clinical efficacy of the current antidepressant therapies.

\section{IDDF2021-ABS-0165 PSYCHOLOGICAL WELL-BEING AND SLEEP QUALITY AMONG HEALTHY STOOL DONORS IN SINGAPORE: A CROSS- SECTIONAL STUDY}

${ }^{1}$ Tzi Shin Toh*, ${ }^{2}$ Jonathan Wei Jie Lee, ${ }^{3}$ Kai Yee Toh, ${ }^{3}$ Jia Pei Ho, ${ }^{3}$ Jeremy Fung Yen Lim, ${ }^{1} \mathrm{Ai}$ Huey Tan, ${ }^{4}$ Chun Wie Chong. 'Division of Neurology, Department of Medicine, Faculty of Medicine, University of Malaya, Kuala Lumpur, Malaysia; ${ }^{2}$ Division of Gastroenterology and Hepatology, National University Hospital, Singapore; ${ }^{3}$ Asian Microbiome Library (AMILI), Singapore; ${ }^{4}$ School of Pharmacy, Monash University Malaysia, Selangor, Malaysia

\subsection{6/gutjnl-2021-IDDF.48}

Background Psychological stress, which is associated with poor sleep quality, has a profound effect on the gut microbiome. To better elucidate the gut microbiome changes associated with psychological stress, we measured levels of stress and sleep quality among the healthy stool donors recruited through a national campaign, and further assessed their relationship with the gut microbiome.

Methods The gut microbial composition of 272 adults residing in Singapore was determined by $16 \mathrm{~S}$ rRNA V3-V4 amplicon sequencing. Stress and distress levels of participants were assessed using the Perceived Stress Scale (PSS) and Kessler Psychological Distress Scale (K10) respectively, while sleep quality was assessed using the Pittsburgh Sleep Quality Index (PSQI). Multivariate statistical data analyses were conducted to explore the link between the gut microbiome, stress, distress and sleep quality.

Results A significant proportion of stool donors exhibited low distress (84.2\%) levels. However, nearly one-third of participants reported high-stress levels (33.5\%) and poor sleep quality (44.8\%) (IDDF2021-ABS-0165 Figure A. Stacked bar plot showing the number of participants under each category of (i) K10, (ii) PSS and (iii) PSQI). The overall variation in the gut microbiome composition amongst stool donors was predominantly dependent on age and body mass index (BMI), rather than psychosocial stress and sleep quality (IDDF2021-ABS-0165 Figure B. PERMANOVA results of main factors in this study). However, four microbial 